\title{
The gene-treatment interaction of paraoxonase- 1 gene polymorphism and statin therapy on insulin secretion in Japanese patients with type 2 diabetes: Fukuoka diabetes registry
}

\author{
Akiko Sumi ${ }^{1}$, Udai Nakamura ${ }^{1}$, Masanori Iwase ${ }^{1,2^{*}}$ (D), Hiroki Fujii ${ }^{3}$, Toshiaki Ohkuma ${ }^{1,4}$, Hitoshi Ide ${ }^{1,5}$, \\ Tamaki Jodai-Kitamura', Yuji Komorita', Masahito Yoshinari ', Yoichiro Hirakawa ${ }^{6}$, Atsushi Hirano ${ }^{1}$, \\ Michiaki Kubo ${ }^{7}$ and Takanari Kitazono ${ }^{1}$
}

\begin{abstract}
Background: Although statins deteriorate glucose metabolism, their glucose-lowering effects have emerged in some situations. Here, we assessed whether these effects are a consequence of statins' interaction with paraoxonase (PON)1 enzyme polymorphism.
\end{abstract}

Methods: Adult Japanese type 2 diabetes patients $(n=3798)$ were enrolled in a cross-sectional study. We used Q192R polymorphism of the PON1 gene as a representative single-nucleotide polymorphism and focused on the effects of the wild-type $\mathrm{Q}$ allele, in an additive manner. For patients with and without statin therapy, the associations of this allele with fasting plasma glucose (FPG), $\mathrm{HbA}_{1 \mathrm{c}}$ C-peptide, $\mathrm{HOMA2}-\% \beta$, and $\mathrm{HOMA2}-\mathrm{IR}$ were investigated separately using a linear regression model, and were compared between groups by testing interactions. Sensitivity analyses were performed using propensity score to further control the imbalance of characteristics between groups.

Results: Among patients with statin therapy, there were linear associations of the number of $Q$ alleles with decreased FPG and $\mathrm{HbA}_{1 \mathrm{c}}$ and with increased serum $\mathrm{C}$ peptide and $\mathrm{HOMA2} \% \beta$ (all $P<0.01$ for trends), while such associations were not observed among those without statin therapy. These differences were statistically significant only for serum $C$ peptide and HOMA2-\% $(P<0.01$ for interactions). These associations remained significant after multiple explanatory variable adjustment. Sensitivity analyses using propensity score showed broad consistency of these associations.

Conclusions: Patients with the Q allele of the PON1 Q192R polymorphism who were treated with statins exhibited improvement in glucose metabolism, especially in insulin secretion, suggesting the importance of genotyping PON1 Q192R to identify those who could benefit from statin therapy.

Keywords: Gene-treatment interaction, PON1 Q192R polymorphism, Statin therapy, Insulin secretion

\footnotetext{
* Correspondence: iwase@intmed2.med.kyushu-u.ac.jp

${ }^{1}$ Department of Medicine and Clinical Science, Graduate School of Medical

Sciences, Kyushu University, 3-1-1 Maidashi, Higashi-ku, Fukuoka 812-8582,

Japan

2Diabetes Center, Hakujyuji Hospital, Fukuoka, Japan

Full list of author information is available at the end of the article
} 


\section{Background}

Hypercholesterolemia is a common comorbidity with diabetes and contributes to the increased risk of cardiovascular disease among affected people [1]. Lipid-lowering with 3-hydroxy-3-methylglutaryl-coenzyme A reductase inhibitors (statins) is an important and recommended therapy to reduce cardiovascular risk and to treat atherosclerosis associated with hypercholesterolemia [1]. Recently, however, several studies have reported adverse effects of statins on glucose metabolism because statins reduce both insulin secretion and insulin sensitivity, and, as a result, deteriorate glycemic control [2-5], although glucose-lowering effects of statins have emerged in some situations [6]. For the improved management of diabetes and lipids, there is a need for a deeper understanding of the glucose-raising effects of statins and the ability to prevent such effects.

Gene-treatment/environment interaction analysis investigates whether the magnitude of the genetic effect estimate differs across the range of treatments or environmental factors [7], and could contribute to revealing the pharmacological mechanism of the enzyme by investigating the association of common genetic variants in the gene encoding that enzyme and treatment factors. 3-Hydroxy-3-methylglutaryl-coenzyme A reductase (HMGCR) is the main enzyme inhibited by statins; its genetic variants have been shown to be associated with increased body weight and the risk of type 2 diabetes among nondiabetic subjects, independently of statin therapy [8]. Paraoxonase (PON) 1 is another enzyme potentially affected by statins [9], which possesses the properties of an antioxidant and an insulin secretagogue [10, 11]. Since the glucose-lowering effects of statins are most pronounced in patients with an improvement in HDL-C upon statin therapy [6] and this improvement was shown to be dependent on the genotype of PON1 [12], it could be hypothesized that the interaction of the PON1 genotype with statins could play a role in changing glucose metabolism in patients treated with statins. However, at present, there is a lack of evidence for such an interaction in patients with type 2 diabetes.

Therefore, in the present study, we performed a quantitative trait interaction analysis testing modifiable effects of statins on the association between PON1 Q192R polymorphism and glycemia, such as fasting plasma glucose, $\mathrm{HbA}_{1 \mathrm{c}}$, insulin secretion measured by serum $\mathrm{C}$-peptide and HOMA2- $\% \beta$, and insulin resistance measured by HOMA2-IR, in Japanese patients with type 2 diabetes.

\section{Methods}

\section{Study participants}

The Fukuoka Diabetes Registry is a multicenter, prospective study designed to investigate the influence of modern therapy on the prognosis of patients with diabetes mellitus in Japan. Patients who regularly attended teaching hospitals authorized by the Japan Diabetes Society or certified diabetes clinics in Fukuoka Prefecture (UMIN Clinical Trial Registry 000002627) [13] were registered between April 2008 and October 2010 if aged $\geq 20$ years. Exclusion criteria were as follows: 1 ) patients with drug-induced diabetes mellitus or receiving corticosteroid therapy; 2) patients who had undergone renal replacement therapy; 3) patients with serious diseases other than diabetes, such as advanced malignancy or decompensated liver cirrhosis; and 4) patients unable to visit diabetologists regularly. Among the 5131 patients registered, after excluding those with type 1 diabetes defined by serum C-peptide level < $0.03 \mathrm{nmol} / \mathrm{l}$ and being on insulin therapy, those who had already eaten breakfast, those with unacceptable levels of plasma glucose $(<3 \mathrm{mmol} / \mathrm{l}$ or $>25 \mathrm{mmol} / \mathrm{l})$ or C-peptide $(<0.2 \mathrm{nmol} / \mathrm{l}$ or $>3.5 \mathrm{nmol} / \mathrm{l})$ for HOMA2-\% $\beta$ [14], and those who had not been genotyped for the PON1 gene, the remaining 3798 patients were enrolled in the present cross-sectional study. The present study was conducted with the approval of Kyushu University Institutional Review Board, and written informed consent was obtained from all of the participants.

\section{Clinical evaluation and laboratory measurements}

The participants completed a self-administered questionnaire to provide data on their smoking habits, duration of diabetes mellitus, alcohol intake, physical activity, family history of diabetes, and past history of cardiovascular disease. Smoking habits and alcohol intake were classified as either current use or not. Metabolic equivalent (MET) hours per week values were calculated using Ainsworth's methods [15]. The participants' medical records were reviewed for all medications, including statin therapy, oral hypoglycemic agents (OHA), and insulin therapy. Body weight and height were measured, and body mass index (BMI) was calculated as weight (kg) divided by height squared $\left(\mathrm{m}^{2}\right)$. Blood pressure was measured with the participants in a sitting position. Hypertension was defined as blood pressure $\geq 140 / 90 \mathrm{mmHg}$ and/or current use of antihypertensive agents. Blood samples were collected via venipuncture under fasting conditions. Hemoglobin $\mathrm{A}_{1 \mathrm{c}}\left(\mathrm{HbA}_{1 \mathrm{c}}\right)$ level was determined by high-performance liquid chromatography (Tosoh Corp., Tokyo, Japan), plasma glucose by the glucose oxidase method, serum C-peptide by a chemiluminescent immunoassay (Kyowa Medex, Tokyo, Japan), and lipid profiles, such as serum total cholesterol, LDL-C, HDL-C, and triglyceride, by enzymatic methods. Beta-cell function and insulin resistance were estimated based on fasting glucose and $\mathrm{C}$-peptide concentrations using the homeostasis model assessment (HOMA) calculator, version 2.2.2 (http:// www.dtu.ox.ac.uk, accessed June 2012), and are expressed as the homeostasis model assessment of $\beta$-cell function (HOMA2-\% $\beta$ ) and the homeostasis model assessment of insulin resistance (HOMA2-IR), respectively. 


\section{Genotyping of PON1 Q192R polymorphism}

The gene encoding the paraoxonase (PON) 1 enzyme has a polymorphism with a reference single-nucleotide polymorphism (SNP) ID number of rs662; this SNP changes amino acid 192 of the PON1 protein. The wildtype, rs662(A), encodes a glutamine $(\mathrm{Q})$, while the variant, rs662(G), encodes an arginine (R).The genotypes of the PON1 Q192R SNP were determined as QQ, QR, or $R R$ using multiplex polymerase chain reaction-based invader assays (Third Wave Technologies, Madison, WI, USA) [16]. In the present analyses, we focused on the effect of the Q-allele, the wild type, compared with the Rallele, as the former confers the high antioxidative effect of the enzyme [9].

\section{Statistical analysis}

The data on C-peptide, HOMA2-\% $\beta$, HOMA2-IR, and triglyceride were log-transformed for the statistical analysis due to their skewed distribution. The chi-square test was used to test the divergence from Hardy-Weinberg equilibrium by determining the difference between observed and expected genotype frequencies from the allele frequencies. The clinical and biochemical characteristics of the participants were compared between those using and not using statins, and between PON1 Q192R genotypes using analysis of variance (ANOVA), chi-square analyses, and Fisher's exact tests.

To investigate a gene-treatment interaction on glycemic parameters, the associations of PON1 Q192R genotypes with fasting plasma glucose (FPG), $\mathrm{HbA}_{1 \mathrm{c}}$, Cpeptide, HOMA2-\% $\beta$, and HOMA2-IR were estimated separately using linear regression models in patients with and without statin therapy, wherein the effects of PON1 Q192R genotypes were assumed to be additive, namely, the number of $\mathrm{Q}$ alleles was counted. These associations were compared using the interaction term of genotypes and use of statins in the relevant statistical model. In multiple explanatory variable analyses, adjustments were made for gender, age, $\mathrm{BMI}, \mathrm{HbA}_{1 \mathrm{c}}$, $\mathrm{OHA}$, insulin therapy, current smoking, current drinking, leisure-time physical activity, and duration of diabetes.

In a nonrandomized study, patients with specific medication might be at higher risk than those without, since there could be a bias in administering medication to severe patients. Such imbalance of background risk might affect the results. Therefore, we performed sensitivity

Table 1 Clinical characteristics in statin-treated or untreated patients with type 2 diabetes mellitus according to PON1 genotype

\begin{tabular}{|c|c|c|c|c|c|c|c|c|}
\hline \multirow{3}{*}{ PON1 Q192R genotype } & \multicolumn{3}{|l|}{ Statin $(-)$} & \multirow{3}{*}{$P$ value } & \multicolumn{3}{|l|}{ Statin $(+)$} & \multirow{3}{*}{$P$ value } \\
\hline & $\overline{Q Q}$ & $\mathrm{QR}$ & $\mathrm{RR}$ & & \multirow{2}{*}{$\begin{array}{l}\mathrm{QQ} \\
N=179,10.7 \%\end{array}$} & \multirow{2}{*}{$\begin{array}{l}\text { QR } \\
N=767,45.7 \%\end{array}$} & \multirow{2}{*}{$\begin{array}{l}\mathrm{RR} \\
N=732,43.6 \%\end{array}$} & \\
\hline & $N=249,11.7 \%$ & $N=887,41.8 \%$ & $N=984,46.4 \%$ & & & & & \\
\hline Male, n (\%) & $167(67.1)$ & $573(64.6)$ & $648(65.9)$ & 0.73 & 78 (43.6) & $371(48.4)$ & $333(45.5)$ & 0.37 \\
\hline Age, years & $65.2 \pm 10.6$ & $64.8 \pm 10.8$ & $64.7 \pm 10.6$ & 0.83 & $66.1 \pm 9.6$ & $66.1 \pm 9.4$ & $65.9 \pm 9.7$ & 0.91 \\
\hline $\mathrm{BMI}, \mathrm{kg} / \mathrm{m}^{2}$ & $23.8 \pm 3.5$ & $23.8 \pm 3.5$ & $24.0 \pm 3.9$ & 0.28 & $24.5 \pm 4.1$ & $24.3 \pm 3.8$ & $24.4 \pm 3.5$ & 0.84 \\
\hline Duration of diabetes, years & $13.8 \pm 10.7$ & $14.4 \pm 10.2$ & $14.4 \pm 10.3$ & 0.68 & $14.3 \pm 10.1$ & $14.6 \pm 9.7$ & $15.4 \pm 9.9$ & 0.17 \\
\hline Current smoker, n (\%) & $52(20.9)$ & $182(20.5)$ & $212(21.5)$ & 0.86 & $27(15.1)$ & $96(12.5)$ & $113(15.4)$ & 0.24 \\
\hline Current drinker, n (\%) & $118(47.4)$ & $398(44.9)$ & $430(43.7)$ & 0.57 & $57(31.8)$ & $244(31.8)$ & $247(33.7)$ & 0.71 \\
\hline $\begin{array}{l}\text { Leisure-time physical activity, } \\
\text { METs.h/w }\end{array}$ & $18.9 \pm 17.5$ & $19.2 \pm 19.2$ & $18.6 \pm 17.9$ & 0.81 & $16.4 \pm 18.5$ & $18.4 \pm 17.5$ & $18.9 \pm 18.7$ & 0.25 \\
\hline $\begin{array}{l}\text { Family history of diabetes, } \\
\mathrm{n}(\%)\end{array}$ & $132(53.0)$ & $491(55.4)$ & $525(53.4)$ & 0.64 & $98(54.8)$ & $446(58.2)$ & $400(54.6)$ & 0.36 \\
\hline Past history of CVD, n (\%) & $47(18.9)$ & $169(19.1)$ & $187(19.0)$ & 1.00 & $58(32.4)$ & $232(30.3)$ & $223(30.5)$ & 0.85 \\
\hline $\mathrm{HDL}$ cholesterol, mmol/l & $1.41 \pm 0.37$ & $1.43 \pm 0.37$ & $1.41 \pm 0.38$ & 0.55 & $1.49 \pm 0.36$ & $1.46 \pm 0.38$ & $1.45 \pm 0.36$ & 0.40 \\
\hline LDL cholesterol, mmol/l & $2.99 \pm 0.75$ & $3.03 \pm 0.71$ & $3.02 \pm 0.75$ & 0.77 & $2.75 \pm 0.68$ & $2.70 \pm 0.61$ & $2.69 \pm 0.62$ & 0.52 \\
\hline $\mathrm{OHA}, \mathrm{n}(\%)$ & $144(57.8)$ & $587(66.2)$ & $645(65.6)$ & 0.043 & $124(69.3)$ & $540(70.4)$ & $525(71.7)$ & 0.76 \\
\hline Sulfonylurea, n (\%) & 91 (36.6) & $397(44.8)$ & $423(43.0)$ & 0.07 & $77(43.0)$ & $390(50.9)$ & $362(49.5)$ & 0.17 \\
\hline Biguanide, n (\%) & $69(27.7)$ & $291(32.8)$ & $346(35.2)$ & 0.08 & $73(40.8)$ & $287(37.4)$ & $291(39.8)$ & 0.55 \\
\hline$a-G l, n(\%)$ & $32(12.9)$ & $105(11.8)$ & $91(9.3)$ & 0.10 & $25(14.0)$ & $109(14.2)$ & $91(12.4)$ & 0.58 \\
\hline Thiazolidine, n (\%) & $20(8.0)$ & $111(12.5)$ & $114(11.6)$ & 0.15 & $36(20.1)$ & $148(19.3)$ & $112(15.3)$ & 0.08 \\
\hline Glinide, n (\%) & $16(6.4)$ & $60(6.8)$ & $58(5.9)$ & 0.74 & $7(3.9)$ & $47(6.1)$ & $41(5.6)$ & 0.51 \\
\hline DPP4-I, n (\%) & $0(0)$ & $4(0.5)$ & $6(0.6)$ & 0.45 & $1(0.56)$ & $2(0.26)$ & $2(0.27)$ & 0.64 \\
\hline Insulin, n (\%) & 57 (22.9) & $178(20.1)$ & $200(20.3)$ & 0.61 & $39(21.8)$ & 145 (18.9) & $154(21.0)$ & 0.50 \\
\hline
\end{tabular}

Data are expressed as mean \pm SD and $\mathrm{n}$ (percentage)

CVD cardiovascular disease, OHA oral hypoglycemic agents, $a$-Gl alpha-glucosidase inhibitor, DPP4-I inhibitors of type 4 dipeptidyl peptidase 
Table 2 Association of PON1 Q192R polymorphism with glycemia, insulin secretion, and sensitivity after stratified analysis by statin therapy

\begin{tabular}{lllllll}
\hline & & $\mathrm{FPG}, \mathrm{mmol} / \mathrm{I}$ & $\mathrm{HbA}_{1,1} \%(\mathrm{mmol} / \mathrm{mol})$ & Logarithm of C-peptide & Logarithm of HOMA2-\% $\beta$ & Logarithm of HOMA2-IR \\
\hline Statin (-) & QQ & $7.75 \pm 2.02$ & $7.23 \pm 0.95(55.5 \pm 10.4)$ & $0.19 \pm 0.39$ & $3.69 \pm 0.45$ & $0.02 \pm 0.43$ \\
& QR & $7.66 \pm 2.04$ & $7.28 \pm 1.03(56.1 \pm 11.2)$ & $0.16 \pm 0.38$ & $3.69 \pm 0.48$ & $-0.02 \pm 0.41$ \\
& RR & $7.73 \pm 2.06$ & $7.35 \pm 1.05(56.8 \pm 11.5)$ & $0.19 \pm 0.39$ & $3.69 \pm 0.50$ & $0.01 \pm 0.41$ \\
& P value & 0.82 & 0.07 & 0.47 & 0.8 & 0.47 \\
Statin (+) & QQ & $7.51 \pm 1.79$ & $7.34 \pm 0.92(56.7 \pm 10.1)^{\#}$ & $0.27 \pm 0.40^{\#}$ & $3.80 \pm 0.49^{\#}$ & $0.09 \pm 0.41$ \\
& QR & $7.53 \pm 1.88^{\#}$ & $7.43 \pm 0.99(57.7 \pm 10.8)$ & $0.22 \pm 0.38$ & $3.76 \pm 0.47^{\#}$ & $0.04 \pm 0.40$ \\
& RR & $7.81 \pm 1.98$ & $7.56 \pm 1.07(59.1 \pm 11.7)$ & $0.19 \pm 0.39$ & $3.67 \pm 0.48$ & $0.02 \pm 0.42$ \\
& P value & 0.0052 & 0.0022 & 0.016 & 0.0001 & 0.082 \\
\hline
\end{tabular}

Data are expressed as mean $\pm \mathrm{SD} ; P$ value refers to simple regression analysis

" $P<0.05$ vs. RR genotype

Interaction of statin therapy with PON1 genotype

analyses using propensity score (PS) to further control the difference of characteristics between patients with and without statins. The PS for the probability of receiving statins was estimated by a multiple explanatory variable logistic regression model using the following clinical variables that might influence the intention to prescribe statins: gender; age; BMI; current smoking and drinking; duration of diabetes; hypertension; past history of stroke, ischemic heart disease, and arteriosclerosis obliterans; antiplatelet therapy; family history of diabetes and hyperlipidemia; leisure-time physical activity; FPG; $\mathrm{HbA}_{1 c}$; LDL-C; HDL-C; logarithm of triglyceride; OHA (sulfonylurea, glinide, DPP4-I, $\alpha$-GI, biguanide, thiazolidine); insulin therapy; fibrate therapy; ezetimibe therapy; ethyl eicosapentate therapy; and the method for controlling blood glucose (diet, OHA, insulin, combination of OHA and insulin). We performed three PS application analyses: one adjusting for PS, one matching patients with and without statin therapy using PS (1:1), and one using the inverse probability of treatment weights (IPTW). Matching for PS was performed in accordance with an optimization protocol using an SAS macro (PSMatch_Multi macro in SAS 9.4) [17], whereby cases and controls were matched if the difference of their scores was equal to or less than 0.01. All analyses were performed using the SAS software package version 9.3 (SAS Institute Inc., Cary, NC, USA). Values of $P<0.05$ were considered to be statistically significant in all analyses.

\section{Results}

Overall, 3798 patients were enrolled in the present study (Additional file 1: Table S1). Their mean age was 65 years and about half of the patients were male. Mean duration of diabetes was 14.6 years. Among the patients, 1678 were being treated with statins (atorvastatin 27.4\%, pravastatin $26.1 \%$, pitavastatin $19.6 \%$, rosuvastatin $17.5 \%$, simvastatin $6.6 \%$, fluvastatin $2.8 \%$ ), and they were older, with a longer duration of diabetes, higher values of $\mathrm{HbA}_{1 \mathrm{c}}$, and a greater frequency of being on oral hypoglycemic therapy than those who were not being treated with statins. The genotype proportions were 0.112 for $\mathrm{QQ}, 0.436$ for $\mathrm{QR}$, and 0.452 for RR (Additional file 2: Table S2), which indicated no divergence from Hardy-Weinberg equilibrium ( $P$ $=0.33$ ). The distributions of genotypes were similar tendency in patients with and without statin therapy $(P=0.055$ for the overall difference) (Table 1). Among all patients (Additional file 2: Table S2) and patients with and without statins (Table 1), the clinical and biochemical characteristics were almost all similar among the PON1 genotypes, except for the rates of use of OHA and sulfonylurea.

In the patients with statin therapy, the number of $\mathrm{Q}$ alleles was associated with decreased values of FPG and $\mathrm{HbA}_{1 \mathrm{c}}$, and with increased C-peptide and HOMA2-\% $\beta(P=0.0052,0.0022,0.016$, and 0.0001 for the trends, respectively), and there was a tendency for a positive association with HOMA2-IR ( $P=0.082$ for trend) (Table 2). In patients without statin therapy, however, no such associations of the number of $\mathrm{Q}$ alleles with any of the glycemic parameters were observed. The difference in such associations between those with and without statins was significant for C-peptide and HOMA2-\% $\beta$, and was marginally insignificant for FPG and HOMA2-IR (Table 2). In the multiple explanatory variable analyses (Fig. 1), there was consistency of these associations, but the heterogeneity in the association of $\mathrm{Q}$ alleles with $\mathrm{C}$-peptide and HOMA2-\% $\%$ remained statistically significant.

As there was a significant difference in background risk between those using and not using statins (Additional file 1: Table S1), we performed sensitivity analyses using PS. The PS estimated here showed acceptable goodness of fit for the discrimination of patients with and without statin therapy (c statistic: 0.747 ). The distributions 


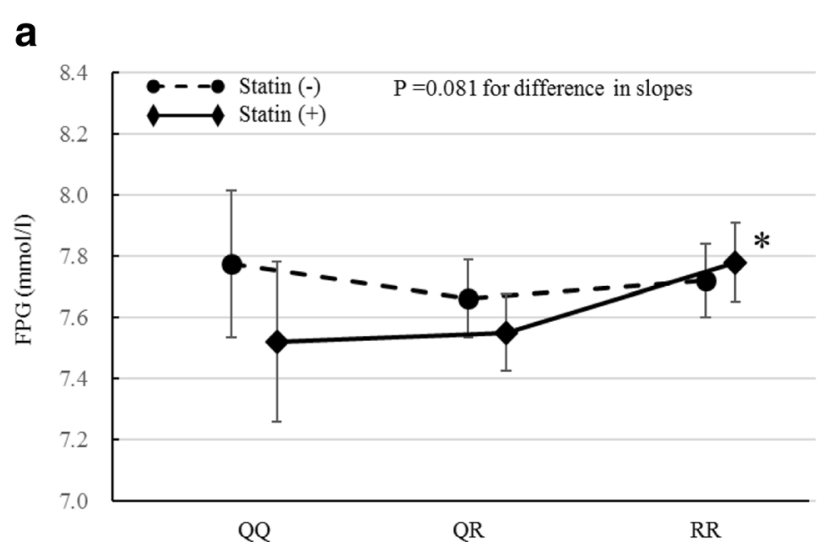

b

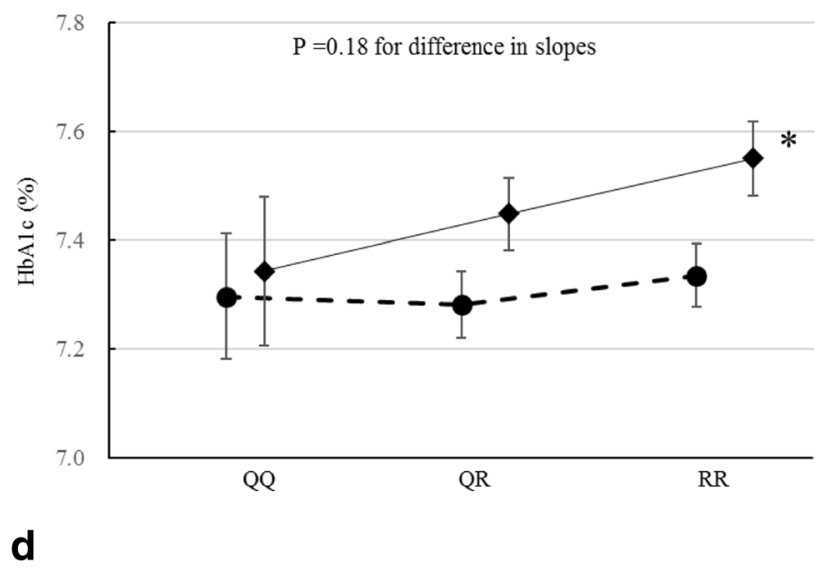

C

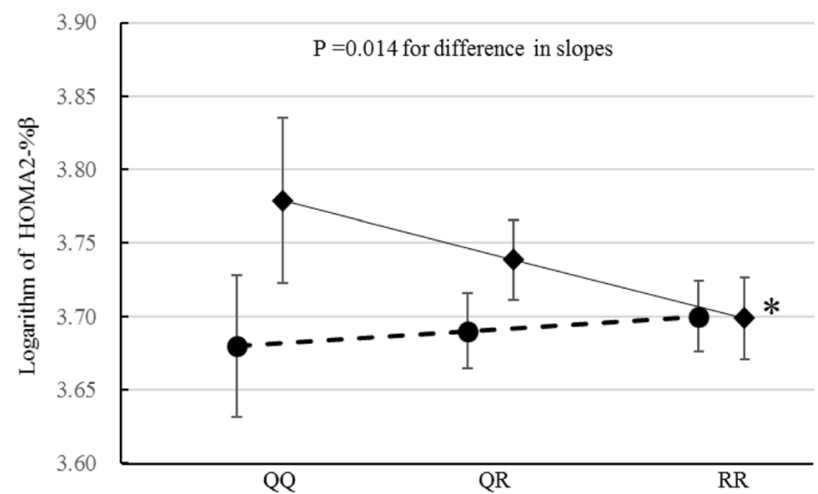

0.10

QQ

QR

RR

Fig. 1 Association of PON1 Q192R polymorphism with FPG (a), HbA1c (b), serum C-peptide (c), and HOMA2-\%ß (d) after stratified analysis by statin therapy. Data are expressed as adjusted mean $(95 \% \mathrm{Cl})$ referring to ANCOVA; $P$ value refers to multiple regression analysis. FPG and HbA 1 : adjusted for gender, age, BMI, smoking, alcohol, leisure-time physical activity, duration of diabetes, SU, glinide, thiazolidine, a-Gl, biguanide, insulin. C-peptide and HOMA2-\%ß: adjusted for gender, age, BMI, smoking, alcohol, leisure-time physical activity, duration of diabetes, HbA ${ }_{1 c}$, SU, glinide, thiazolidine, $\mathrm{a}-\mathrm{Gl}$, biguanide, insulin. * $P<0.05$ for trend in the group with statins

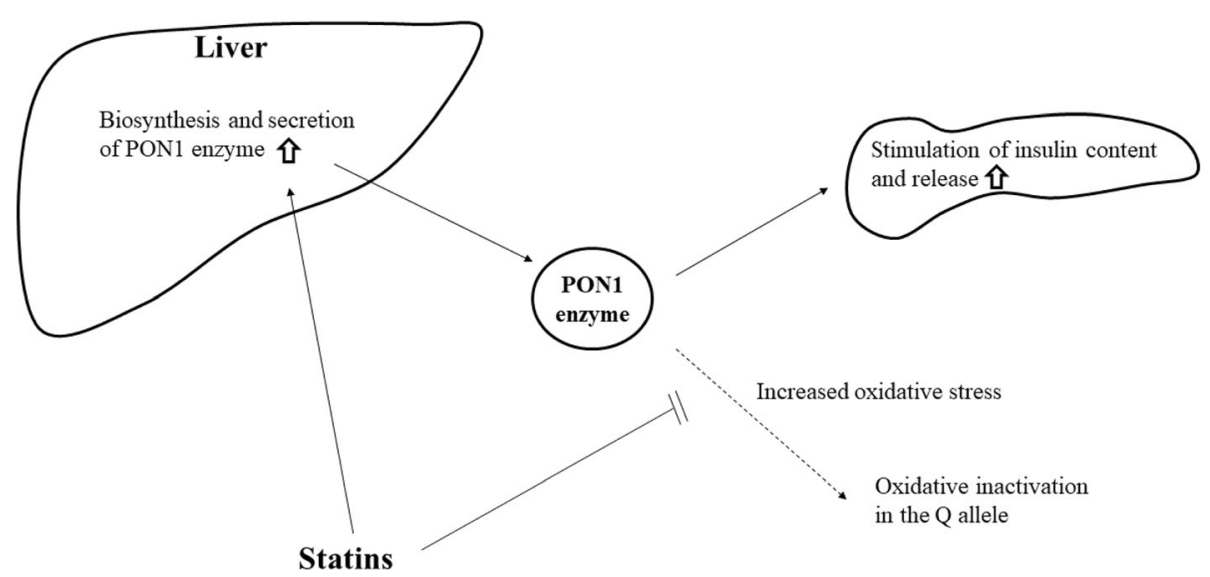

Fig. 2 Proposed model for the interaction of PON1 Q192R polymorphism and statins on insulin secretion 
of the PS in the groups using and not using statins almost overlapped (Additional file 3: Figure S1), and the adjustment for and matching with PS could be reliably performed. In the matched design analyses, patients' characteristics at baseline were almost balanced between the groups with and without statins (Additional file 4: Table S3). All of the three sensitivity analyses showed similar results, with positive linear associations of the number of $Q$ alleles with C-peptide and HOMA2-\% $\beta$ being consistently found across the analyses (Additional file 5: Figure S2, Additional file 6: Figure S3 and Additional file 7: Figure S4).

\section{Discussion}

This is the first study to report the gene-treatment interaction of the PON1 Q192R polymorphism and statin therapy on insulin secretion among patients with type 2 diabetes. We demonstrated that, when diabetic patients were treated with statins, the number of $\mathrm{Q}$ alleles of the PON1 Q192R polymorphism, the wild-type allele, was associated with increased insulin secretion, while no such association was found among those who were not treated with statins. There were similar favorable influences of the $\mathrm{Q}$ allele on fasting plasma glucose and $\mathrm{HbA}_{1 \mathrm{c}}$, although the differences of such effects between the groups with and without statins were not statistically significant.

The underlying mechanism of how PON1 Q192R polymorphism and statins interact on insulin secretion in patients with type 2 diabetes remains unclear. One possible explanation is as follows. The PON1 enzyme has the ability to reduce oxidative stress [9] and to increase insulin secretion [10], and the PON1 Q192R polymorphism has the most significant impact on the enzyme's activity, with the $\mathrm{Q}$ allele being associated with greater enzymatic efficiency [18]. Among patients with type 2 diabetes [19] and with diseases associated with oxidative stress [8], PON1 activity was found to be low, probably due to increased oxidative stress inactivating the PON1 enzyme [20]. Since statins have antioxidant effects and activate the biosynthesis and secretion of the PON1 enzyme in the liver, they may contribute to protecting the PON1 enzyme against inactivation and maintaining its functions [8]. It was also reported that QQ homozygotes showed greater loss of enzyme activity [21] with aging. The enzyme in subjects with this genotype might thus be more vulnerable to such deterioration than that in those with other genotypes and the use of statins could confer their protective effects in this genotype. This possible mechanism is illustrated in Fig. 2. Statins may increase the biosynthesis and secretion of the PON1 enzyme in the liver, and protect the PON1 enzyme from being inactivated by increased oxidative stress. Consequently, insulin secretion may increase in the $\mathrm{Q}$ allele carriers.
Some limitations of this study are a lack of the genotyping of other polymorphisms of the PON1 gene and the lack of availability of data on the serum concentrations and activities of PON1. However, among the major PON1 polymorphisms, only the Q192R polymorphism has been reported to determine the antioxidant potential of PON1 [9], so the influence of this limitation might be small.

\section{Conclusions}

To the best of our knowledge, this is the first study to investigate the possible glucose-lowering effects of PON1 Q192R genotypes associated with statin therapy. These findings could partly delineate the complex influence of statins on glucose metabolism. We suggest that PON1 Q192R genotyping could identify individuals who would benefit from statin therapy for both lipid- and glucose-lowering. Research considering other types of polymorphism in the PON1 gene, and serum concentrations and activities of PON1 would be helpful in pursuit of the mutual effects of statins and PON1 polymorphism on glucose metabolism.

\section{Additional files}

\begin{abstract}
Additional file 1: Table S1. Clinical characteristics according to statin therapy. Data are expressed as mean \pm SD, median (interquartile), and $n$ (percentage). CVD: cardiovascular disease, OHA: oral hypoglycemic agents, a-Gl: alpha-glucosidase inhibitor, DPP4-I: inhibitors of type 4 dipeptidyl peptidase.* log-transformed for the statistical analysis. (DOCX $15 \mathrm{~kb}$ )
\end{abstract}

Additional file 2: Table S2. Clinical characteristics according to PON1 genotype. Data are expressed as mean \pm SD, median (interquartile), and $n$ (percentage). CVD: cardiovascular disease, $\mathrm{OHA}$ : oral hypoglycemic agents, a-Gl: alpha-glucosidase inhibitor, DPP4-I: inhibitors of type 4 dipeptidyl

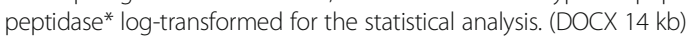

Additional file 3: Figure S1. Overlap of the distributions of the PS in the groups with and without statins. The band and cross marks inside the boxes represent the median and mean values, respectively. The lower and upper edges of the boxes represent the 25th and 75th percentiles, respectively. The upper and lower lines outside the boxes represent minimum and maximum values. (DOCX $77 \mathrm{~kb}$ )

Additional file 4: Table S3. Clinical characteristics according to statin therapy (after 1:1 matching). Data are expressed as mean \pm SD, median (interquartile), and n (percentage). CVD: cardiovascular disease, OHA: oral hypoglycemic agents, a-Gl: alpha-glucosidase inhibitor, DPP4-I: inhibitors of type 4 dipeptidyl peptidase.* log-transformed for the statistical analysis. (DOCX $14 \mathrm{~kb}$ )

Additional file 5: Figure S2. Association of PON1 Q192R polymorphism with FPG, HbA1C, C peptide, and HOMA2-\% $\beta$ after stratified analysis by statin therapy (PS as a covariate). Data are expressed as adjusted mean $(95 \% \mathrm{Cl})$ referring to ANCOVA; $P$ value refers to multiple regression analysis. ${ }^{*} P<0.05$ for trend. (DOCX 67 kb)

Additional file 6: Figure S3. Association of PON1 Q192R polymorphism with FPG, HbA1c, C peptide, and HOMA2-\% $\beta$ after stratified analysis by statin therapy (after 1:1 matching). Data are expressed as mean (SD) referring to ANCOVA; $P$ value refers to multiple regression analysis. ${ }^{*} P<0.05$ for trend. (DOCX $72 \mathrm{~kb}$ )

Additional file 7: Figure S4. Association of PON1 Q192R polymorphism with FPG, HbA1c, C peptide, and $\mathrm{HOMA2-} \% \beta$ after stratified analysis by statin therapy (IPTW). Data are expressed as adjusted mean ( $95 \% \mathrm{Cl}$ ) referring to ANCOVA; $P$ value refers to multiple regression analysis. ${ }^{*} P<0.05$ for trend. (DOCX $68 \mathrm{~kb})$ 


\section{Abbreviations}

CVD: Cardiovascular disease; DPP4-I: Inhibitors of type 4 dipeptidyl peptidase; FPG: Fasting plasma glucose; HOMA2-\% $\beta$ : Homeostasis model assessment $\beta$ cell function; HOMA2-IR: Homeostasis model assessment insulin resistance; IPTW: Inverse probability of treatment weights; OHA: Oral hypoglycemic agents; PON1: Paraoxonase-1; PS: Propensity scores; SNP: Single-nucleotide polymorphism; a-Gl: Alpha-glucosidase inhibitor

\begin{abstract}
Acknowledgments
The authors thank Drs. Yutaka Kiyohara, Yasufumi Doi, Toshiharu Ninomiya, Shigenobu Kanba, Dongchon Kang, Shuzo Kumagai, Shinako Kaizu, Chisa Matsumoto (Kyushu University), Nobutaka Tsutsu, Nobuhiro Sasaki (Fukuoka Red Cross Hospital), Kiyohide Nunoi, Yuichi Sato, Yuji Uchizono, Ayumi Yamauchi, Kaori Itoh, Chie Miyakawa (St. Mary's Hospital), Sakae Nohara Hirofumi Imoto, Kazushi Amano, Chie Kitaoka (Steel Memorial Yawata Hospital), Daisuke Gotoh, Toshitaka Himeno, Masae Toyonaga (Kyushu Central Hospital), Noriyasu Shinohara, Ayako Tsutsumi (Fukuoka Higashi Medical Centre), Yasuhiro Idewaki, Masahiro Nakano, Mina Matsuo, Shoko Morimoto, Tomoko Hyodo (Hakujyuji Hospital), Masae Minami (Clinic Minami Masae), Miya Wada (Wada Miya Naika Clinic), Yoshifumi Yokomizo (Yokomizo Naika Clinic), Yohei Kikuchi and Masanori Kikuchi (Kikuchi Naika Clinic), Riku Nomiyama (Suzuki Naika Clinic), Shin Nakamura (Nakamura Naika Clinic), Kenji Tashiro (Oshima Eye Hospital), Mototaka Yoshinari (Yoshinari Naika Clinic), Kojiro Ichikawa (Fukutsu Naika Clinic), and Teruo Omae (Hisayama Research Institute For Lifestyle Diseases); clinical research coordinators Chiho Ohba, Yumi Ono (Hisayama Research Institute For Lifestyle Diseases), and Kayoko Sekioka (Kyushu University); and administrative staff Tomoko Matake (Hisayama Research Institute For Lifestyle Diseases) and Junko Ishimatsu (Kyushu University).
\end{abstract}

\section{Funding}

This work was supported, in part, by the Japan Society for the Promotion of Science KAKENHI (grant numbers 23,249,037 and 23,659,353 to M.I., and 16 K00861 to H.F.) from the Ministry of Education, Culture, Sports, Science and Technology of Japan.

\section{Availability of data and materials}

The datasets used and/or analysed during the current study are available from corresponding author on reasonable request.

\section{Authors' contributions}

AS, UN and MI were responsible for the study concept and design. AS, HF and YH conducted the analyses. TO, HI, YJ-K, YK, MY and TK helped with interpreting the data and contributed to the discussion. AS, YH and $\mathrm{Ml}$ drafted the manuscript. AH and MK analyzed SNP. All authors participated in critically revising the manuscript and approved the final version.

\section{Ethics approval and consent to participate}

This study was approved by the Kyushu University Institutional Review Board (UMIN Clinical Trial Registry 000002627), and written informed consent was obtained from all participants.

\section{Consent for publication}

Not applicable.

\section{Competing interests}

The authors declare that they have no competing interests.

\section{Publisher's Note}

Springer Nature remains neutral with regard to jurisdictional claims in published maps and institutional affiliations.

\section{Author details}

'Department of Medicine and Clinical Science, Graduate School of Medical Sciences, Kyushu University, 3-1-1 Maidashi, Higashi-ku, Fukuoka 812-8582, Japan. ${ }^{2}$ Diabetes Center, Hakujyuji Hospital, Fukuoka, Japan. ${ }^{3}$ Centre for Cohort Studies, Graduate School of Medical Sciences Kyushu University, Fukuoka, Japan. ${ }^{4}$ The George Institute for Global Health University of Sydney, Sydney, Australia. ${ }^{5}$ Division of General Internal Medicine, School of Oral Health Science, Kyushu Dental University, Kitakyushu, Japan. ${ }^{6}$ Epidemiology and Public Health, Graduate School of Medical Sciences, Kyushu University,
Fukuoka, Japan. ${ }^{7}$ Center for Integrative Medical Sciences, RIKEN, Yokohama, Japan.

Received: 1 March 2017 Accepted: 1 December 2017

Published online: 12 December 2017

\section{References}

1. Diagnosis and Treatment of Diseases of Lipid and Lipoprotein Metabolism in Adults and Children: Diabetes and Dyslipidemia [article online], 2000-2016. Available from https:/www.ncbi.nlm.nih.gov/books/NBK305900/. Accessed 26 Dec 2016.

2. Vallejo-Vaz AJ, Kondapally Seshasai SR, Kurogi K, Michishita I, Nozue T, Sugiyama S, Tsimikas S, Yoshida H, Ray KK. Effect of pitavastatin on glucose, $\mathrm{HbA}_{1 c}$ and incident diabetes: a meta-analysis of randomized controlled clinical trials in individuals without diabetes. Atherosclerosis. 2015; 241:409-18.

3. Cederberg H, Stančáková A, Yaluri N. Increased risk of diabetes with statin therapy is associated with impaired insulin sensitivity and insulin secretion: a 6 year follow-up study of the METSIM cohort. Diabetologia. 2015; 58:1109-17.

4. Ishikawa M, Namiki A, Kubota T, Yajima S, Fukazawa M, Moroi M, Sugi K. Effect of pravastatin and atorvastatin on glucose metabolism in nondiabetic patients with hypercholesterolemia. Intern Med. 2006:45:51-5.

5. Koh KK, Quon MJ, Han SH, Lee Y, Kim SJ, Shin EK. Atorvastatin causes insulin resistance and increases ambient glycemia in hypercholesterolemic patients. J Am Coll Cardiol. 2010:55:1209-16.

6. Koren-Gluzer M, Aviram M, Meilin E, Hayek T. The antioxidant HDLassociated paraoxonase-1 (PON1) attenuates diabetes development and stimulates $\beta$-cell insulin release. Atherosclerosis. 2011;219:510-8.

7. Franks PW, Pearson E, Florez JC. Gene-environment and gene-treatment interactions in type 2 diabetes: progress, pitfalls, and prospects. Diabetes Care. 2013;36:1413-21.

8. Swerdlow DI, Preiss D, Kuchenbaecker KB, HMG-coenzyme A. Reductase inhibition, type 2 diabetes, and bodyweight: evidence from genetic analysis and randomised trials. Lancet. 2015;385:351-61.

9. Ferretti G, Bacchetti T, Sahebkar A. Effect of statin therapy on paraoxonase-1 status: a systematic review and meta-analysis of 25 clinical trials. Prog Lipid Res. 2015;60:50-73.

10. Mackness M, Mackness B. Human paraoxonase-1 (PON1): gene structure and expression, promiscuous activities and multiple physiological roles. Gene. 2015:567:12-21.

11. Kakuda H, Matoba M, Nakatoh H, Nagao S, Takekoshi N. Effects of change in high-density lipoprotein cholesterol by statin switching on glucose metabolism and renal function in hypercholesterolemia. J Clin Lipidol. 2015; 9:709-15.

12. de Souza JA, Menin A, Lima LO, Smiderle L, Hutz MH, Van Der Sand CR, Van Der Sand LC, Ferreira ME, Pires RC, Almeida S, Fiegenbaum M. PON1 polymorphisms are predictors of ability to attain HDL-C goals in statintreated patients. Clin Biochem. 2015;48:1039-44.

13. Ohkuma T, Fujii H, Iwase M. Impact of eating rate on obesity and cardiovascular risk factors according to glucose tolerance status: the Fukuoka diabetes registry and the Hisayama study. Diabetologia. 2013;56:70-7.

14. Wallace TM, Levy JC, Matthews DR. Use and abuse of HOMA modeling. Diabetes Care. 2004;12:1487-95.

15. Ainsworth BE, Haskell WL, Whitt MC. Compendium of physical activities: an update of activity codes and MET intensities. Med Sci Sports Exerc. 2000;32: S498-504.

16. Ohnishi Y, Tanaka T, Ozaki K, Yamada R, Suzuki H, Nakamura YA. Highthroughput SNP typing system for genome-wide association studies. J Hum Genet. 2001;46:471-7.

17. Healthcare Analytics: A general SAS ${ }^{\star}$ macro to implement optimal N:1 propensity score matching within a maximum radius [article online], 2015. Available from http://www.pharmasug.org/2015-proceedings.html. Accessed 26 Dec 2016.

18. Deakin SP, James RW. Genetic and environmental factors modulating serum concentrations and activities of the antioxidant enzyme paraoxonase-1. Clin Sci. 2004;107:435-47.

19. Belwalker GJ, Jagtap PE, Nagane NS, Dhonde S. PON1 activity in newly detected type 2 diabetes mellitus patients with and without hypertension. Int J Pharm Bio Sci. 2013;4:1298-302. 
20. Aviram M, Rosenblat M, Billecke S, Erogul J, Sorenson R, Bisgaier CL, Newton RS, La DB. Human serum paraoxonase (PON1) is inactivated by oxidised low density lipoprotein and preserved by antioxidants. Free Rad Biol Med. 1999; 26:892-904.

21. Sentí M, Tomás M, Vila J, Marrugat J, Elosua R, Sala J, Masiá R. Relationship of age-related myocardial infarction risk and Gln/Arg 192 variants of the human paraoxonase1 gene: the REGICOR study. Atherosclerosis. 2001;156: 443-9.

Submit your next manuscript to BioMed Central and we will help you at every step:

- We accept pre-submission inquiries

- Our selector tool helps you to find the most relevant journal

- We provide round the clock customer support

- Convenient online submission

- Thorough peer review

- Inclusion in PubMed and all major indexing services

- Maximum visibility for your research

Submit your manuscript at www.biomedcentral.com/submit 